\title{
Influence of Uniaxial Pressure on the Refractive Properties of Rubidium Tetrachlorine Zincanate Crystals
}

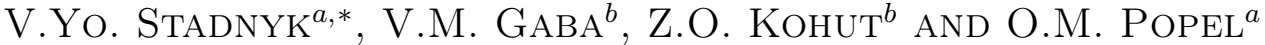 \\ ${ }^{a}$ Ivan Franko Lviv National University, 8 Kyrylo and Mefodiy St., 79005 Lviv, Ukraine \\ ${ }^{b}$ Lviv Polytechnic National University, 12 Bandera St., 79013 Lviv, Ukraine
}

\begin{abstract}
The effect of uniaxial pressure $\left(\sigma_{\mathrm{m}} \leq 200 \mathrm{bar}\right)$ on the spectral $(300-800 \mathrm{~nm})$ and temperature $(77-300 \mathrm{~K})$ dependences of the refractive indices of $\mathrm{Rb}_{2} \mathrm{ZnCl}_{4}$ crystals are investigated. The baric dependences of the electron polarizability, specific refraction and the parameters involved in the Sellmeier formula are calculated.
\end{abstract}

PACS numbers: 61.44.Fw, 77.84.Fa, 78.20.Ci

\section{Introduction}

$\mathrm{Rb}_{2} \mathrm{ZnCl}_{4}$ crystals (TCZR) belong to a known type of ferroelectrics that possess an incommensurate phase (ICP). They undergo two successive phase transitions (PT): a second-order transition $\left(T_{\mathrm{i}}=302 \mathrm{~K}\right)$ from the paraphase (the space group Pnam) to the ICP (the modulation wave vector $\left.q=\left(\frac{1}{3}-\delta\right) c^{*}\right)$, and a first-order one $\left(T_{\mathrm{c}}=192 \mathrm{~K}\right)$ from the ICP to the improper ferroelectric commensurate phase (CP) (the space group $P n a_{1} 2$ ), with the spontaneous polarization $P_{\mathrm{s}}$ arising along $X$ direction $\boldsymbol{P}_{\mathrm{c}} \| \boldsymbol{a}$ [1-3].

As the temperature decreases inside the ICP and the CP-ICP point $T_{\mathrm{c}}$ is reached, the modulation period approaches the commensurate value and the structure becomes domain-like. Just above $T_{\mathrm{c}}$ the crystal consists of locally commensurate regions separated by domain walls (phase solitons) [4,5]. The structure changes from the $\mathrm{CP}$ to the ICP and vice versa are thought to be achieved through nucleation or annihilation of those solitons.

The high-temperature phase in TCZR has a structure of $\beta-\mathrm{K}_{2} \mathrm{SO}_{4}$ type. In the $\mathrm{CP}$ phase, the lattice parameter is tripled along the $c$ axis as compared with that in the paraphase.

Optical investigations of the TCZR crystal (the temperature and spectral changes in the refractive indices and the birefringence, along with the temperature dependences of combined piezooptic coefficients) have shown anomalies at the PT's [7-9].

Despite a long-standing interest in the TCZR, the effect of uniaxial pressures on their optical characteristics and their behaviour near the points of PT's has not been studied yet. Such the pressures generally do not change crystal symmetry, but make it possible to affect selec-

* corresponding author; e-mail: vasylstadnyk@ukr.net tively some groups of bonds and the corresponding structural units, thus providing additional information for the analysis of PT's mechanisms. When studying the refractive indices and the birefringence, one can derive useful information about the structure and physical properties of the crystals.

The purpose of the present work is to study the influence of uniaxial mechanical pressure along the principal $X, Y$ and $Z$ directions and the corresponding bisectors upon spectral and temperature dependences of the refractive indices $n_{i}$ of TCZR crystals, for the case of light propagating in the $X, Y$ and $Z$ directions.

\section{Experimental procedure}

Single crystals of TCZR were grown using a slow evaporation of aqueous solution of stoichiometric mixture of $\mathrm{RbCl}$ and $\mathrm{ZnCl}_{2}(310 \mathrm{~K})$. Crystal plates cut perpendicular to the principal axes were used for our experiments. Typical dimensions of the plates were $100 \mathrm{~mm}^{2}$ in area and $1 \mathrm{~mm}$ thickness. No twinning was visible under microscopic observation. The samples were placed into a cryostat filled with helium gas which enabled the temperature to be kept uniform to within $0.01 \mathrm{~K}$.

Dispersion $n_{i}(\lambda)$ in the spectral range of $300-800 \mathrm{~nm}$ was studied, following from changes in the interference pattern for the polarized light, which was detected using a DFS-8 spectral device with a modernized input unit. If white light passes through such a system, the interference pattern arises in the focal plane of a spectrometer. The minima satisfy the condition

$$
n_{i}=1+\frac{k \lambda}{d}
$$

where $k$ means the order of the interference, $d$ the sample thickness along the ray, and $n_{i}$ the refractive index for the principal direction $i$.

Direct studies of the refractive indices of samples under pressure represent a difficult technical problem. Here, the 
baric changes in the refractive indices $n_{i}$ of TZCR crystal were analyzed through determination of the piezooptic constants $\pi_{i \mathrm{~m}}$ and subsequent calculations of $n_{i}(\sigma)$ from the formula

$$
n_{i}(\lambda, T)=n_{i 0}(\lambda, T)-\frac{1}{2} \pi_{i \mathrm{~m}}(\lambda, T) \times \sigma_{\mathrm{m}} n_{i 0}^{3}(\lambda, T),
$$

where $n_{i 0}$ is the refractive index for mechanically free crystal.

The above technique makes it possible to investigate the behaviour of the refractive indices under various pressures (especially the changes in $n_{i}$ under $\sigma_{i}$ ) for wide spectral and temperature regions. The well-known direct interference optical method [9] allows obtaining the corresponding dependences for one wavelength only. Moreover, it is a very difficult for practical implementation.

\section{Experimental results and discussion}

Figure 1 shows baric dependences of the principal refractive indices $n_{i}$ of TCZR at room temperature for different pressure directions. It can be seen that uniaxial compression increases the refractive indices: under the pressure $\sigma_{\mathrm{m}} \sim 200$ bar, $n_{i}$ increases by $(4-5) \times 10^{-4}$ on the average $\left(\partial n_{i} / \partial \sigma \sim 2.2 \times 10^{-6} \mathrm{bar}^{-1}\right)$.

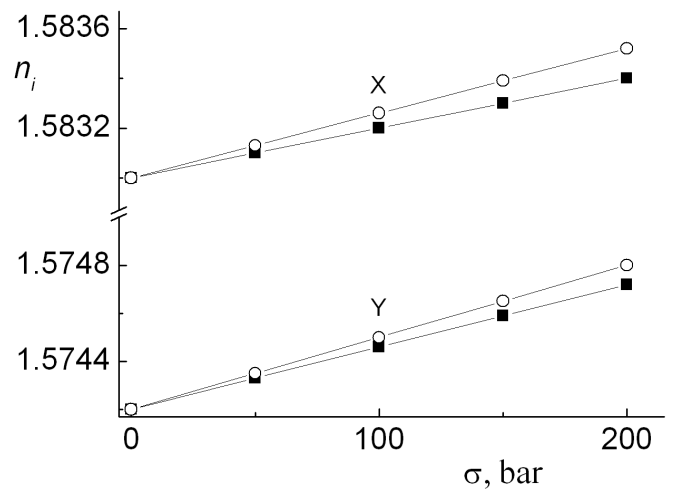

Fig. 1. Baric dependences of the refractive indices of $\mathrm{Rb}_{2} \mathrm{ZnCl}_{4}$ crystals at room temperature for $\lambda=500 \mathrm{~nm}$ : open circles - the pressures $\sigma_{y}$ and full squares - the pressures $\sigma_{z}$.

Figure 2 shows dispersion of the refractive indices $n_{i}$ at room temperature for mechanically free TCZR crystals. One can see that the dispersion $n_{i}(\lambda)$ is normal in the spectral region of $250-800 \mathrm{~nm}$ and sharply increases approaching the absorption edge $\left(\mathrm{d} n_{i} / \mathrm{d} \lambda=\right.$ $-(11.2-12.7) \times 10^{-5} \mathrm{~nm}^{-1}$ near $500 \mathrm{~nm}$ and $\mathrm{d} n_{i} / \mathrm{d} \lambda=$ $-(68.5-72.8) \times 10^{-5} \mathrm{~nm}^{-1}$ near $\left.300 \mathrm{~nm}\right)$. It was shown that uniaxial pressures change the parameter of dispersion $\mathrm{d} n_{i} / \mathrm{d} \lambda, \mathrm{d} n_{x} / \mathrm{d} \lambda=-72.8 \times 10^{-5}$ and $-70.9 \times 10^{-5}$, $\mathrm{d} n_{y} / \mathrm{d} \lambda=-69.7 \times 10^{-5}$ and $-67.9 \times 10^{-5} \mathrm{~nm}^{-1}$ near $\lambda=$ $300 \mathrm{~nm}$ (respectively, for mechanically free sample and for the sample under the uniaxial pressure $\sigma_{z}=200 \mathrm{bar}$ ).

Let us consider the relationship between $n_{i}(\lambda, \sigma)$ and other characteristics of the crystal, given by the Sellmeier

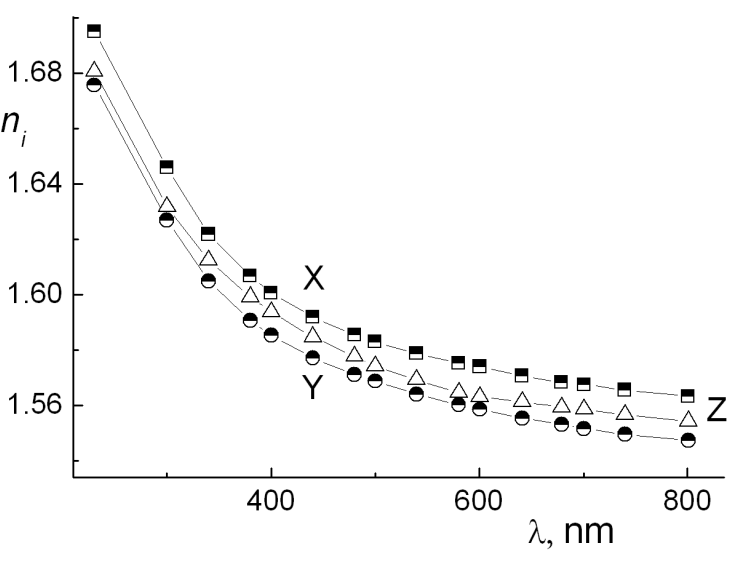

Fig. 2. Dispersion of the refractive indices $n_{i}$ for mechanically free $\mathrm{Rb}_{2} \mathrm{ZnCl}_{4}$ crystals at room temperature. Open symbols correspond to unstressed sample, whereas half-opened and solid ones to mechanically stressed one.

dispersion formula and the Lorentz-Lorenz formula describing the refraction $R$ and the electron polarizability $\alpha$ :

$$
\begin{aligned}
& n_{i}^{2}=1+\frac{B_{1 i} \lambda^{2} \lambda_{01}^{2}}{\lambda^{2}-\lambda_{01}^{2}}+\frac{B_{2 i} \lambda^{2} \lambda_{02}^{2}}{\lambda_{02}^{2}-\lambda^{2}}, \\
& \alpha=\frac{3}{4 \pi N} \frac{n^{2}-1}{n^{2}+2}, \\
& R=4 \pi / 3 N_{\mathrm{A}} \alpha=\frac{\mu}{\rho} \frac{n^{2}-1}{n^{2}+2} .
\end{aligned}
$$

Here $N$ is the number of particles per unit volume, $N_{\mathrm{A}}$ the Avogadro's number; $\mu$ the molar mass, $\rho$ the crystal density, and $\lambda_{01}$ and $\lambda_{02}$ are the peak wavelengths of the absorption bands for ultraviolet (UV) and infrared (IR) oscillators, respectively.

Using the experimental $n_{i}(\lambda, \sigma)$ dependences and the relations $(3)-(5)$, we have calculated the parameters collected in Table. The data presented in Table indicate that the uniaxial pressures lead mainly to decrease in the effective UV oscillator strength and to a red shift of the effective center of the UV absorption band. For the pressure $\sigma_{x}$, this shift is the largest (on the average, we have $\left.\partial \lambda_{0 i} / \partial \sigma_{\mathrm{m}}=(2-4) \times 10^{-3} \mathrm{~nm} / \mathrm{bar}\right)$. It is also seen from Table that the pressures up to 200 bar increase $\alpha_{i}$ ( (1-4) $\times 10^{-26} \mathrm{~cm}^{-3}$, on the average).

Thus, the increase of absolute refractive indices $n_{i}$ is caused by diminishing effective strength of the UV oscillators and displacement of the effective center of UV absorption band towards long-wave region. Higher sensibility of $n_{i}(T)$ dependences to the stresses is observed in the close region of the ICP.

On our mind, this is caused by peculiarities of interaction between the modulated structure and the uniaxial deformation. A comparison of the refractive parameters of $\mathrm{Rb}_{2} \mathrm{ZnCl}_{4}$ and $\mathrm{K}_{2} \mathrm{ZnCl}_{4}$ crystals shows the following. The $\mathrm{Rb}^{+} \rightarrow \mathrm{K}^{+}$replacement does lead to substantial changes in $n_{i}\left(\right.$ an increase amounts to $\left.(7-12) \times 10^{-3}\right)$ 
TABLE

Baric dependences of electronic polarizability $\alpha_{i}$, specific refraction $R_{i}(\lambda=500 \mathrm{~nm})$ and the parameters of effective oscillators appearing in the Selmeier formula for $\mathrm{Rb}_{2} \mathrm{ZnCl}_{4}$ crystal at $T=294 \mathrm{~K} . \sigma_{\mathrm{m}}=0$ bar, $\sigma_{x}=\sigma_{y}=\sigma_{z}=200$ bar.

\begin{tabular}{l|c|c|c|c}
\hline \hline \multicolumn{1}{c|}{ Parameters } & $\sigma_{\mathrm{m}}$ & $\sigma_{x}$ & $\sigma_{y}$ & $\sigma_{z}$ \\
\hline$\alpha_{x} \times 10^{24}\left[\mathrm{~cm}^{3}\right]$ & 23.22 & 23.27 & 23.26 & 23.25 \\
$\alpha_{y} \times 10^{24}\left[\mathrm{~cm}^{3}\right]$ & 22.76 & 22.82 & 22.83 & 22.81 \\
$\alpha_{z} \times 10^{24}\left[\mathrm{~cm}^{3}\right]$ & 22.94 & 23.00 & 23.01 & 22.99 \\
$R_{x}\left[\mathrm{~cm}^{3}\right]$ & 58.55 & 58.68 & 58.65 & 58.67 \\
$R_{y}\left[\mathrm{~cm}^{3}\right]$ & 57.38 & 57.71 & 57.69 & 57.68 \\
$R_{z}\left[\mathrm{~cm}^{3}\right]$ & 57.83 & 57.99 & 57.96 & 57.95 \\
$\lambda_{0 x}[\mathrm{~nm}]$ & 125.081 & 125.123 & 125.122 & 122.121 \\
$\lambda_{0 y}[\mathrm{~nm}]$ & 122.443 & 122.447 & 122.448 & 122.445 \\
$\lambda_{0 z}[\mathrm{~nm}]$ & 122.963 & 122.967 & 122.968 & 122.966 \\
$B_{1 x} \times 10^{-6}\left[\mathrm{~nm}^{-2}\right]$ & 90.20 & 90.24 & 90.25 & 90.23 \\
$B_{1 y} \times 10^{-6}\left[\mathrm{~nm}^{-2}\right]$ & 91.54 & 90.58 & 90.59 & 90.57 \\
$B_{1 z} \times 10^{-6}\left[\mathrm{~nm}^{-2}\right]$ & 91.49 & 91.53 & 91.54 & 91.52
\end{tabular}

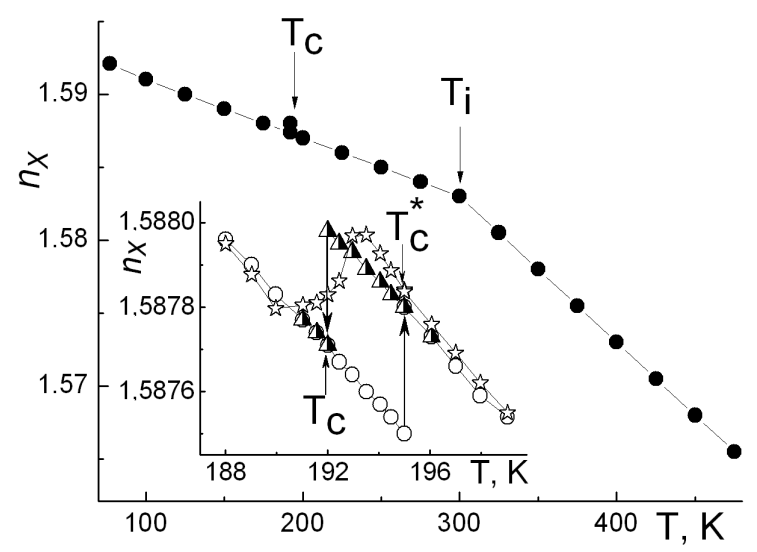

Fig. 3. Temperature dependences of the refractive indices of $\mathrm{Rb}_{2} \mathrm{ZnCl}_{4}$ annealed crystals at $\lambda=500 \mathrm{~nm}$. The inset show temperature dependences of $n_{x}$ near the ICP-CP phase transition. $T_{\mathrm{c}}$ - the temperature of ICP-CP phase transition at cooling (open circles), $T_{\mathrm{c}}^{*}$ - the temperature of $\mathrm{ICP}-\mathrm{CP}$ phase transition at heating (triangle points); star points - dependences of $n_{x}$ near ICP-CP phase transition for as-grown crystal.

with the centers of gravity of the UV oscillators shifted towards shorter $\left(\Delta \lambda_{0 i} \sim 3-5 \mathrm{~nm}\right)$ wavelengths.

The $\lambda_{01 i}$ shift is accompanied with differing changes in the strength of effective UV oscillators. Temperature dependences of $n_{i}(T)$ for $\lambda=500 \mathrm{~nm}$ are shown in Fig. 3. One can see that the principal refractive indices increase almost linearly with decreasing temperature inside the paraphase: $\mathrm{d} n_{x} / \mathrm{d} T=-9.1 \times 10^{-4} \mathrm{~K}^{-1}$, $\mathrm{d} n_{y} / \mathrm{d} T=7.3 \times 10^{-4} \mathrm{~K}^{-1}$ and $\mathrm{d} n_{z} / \mathrm{d} T=7.7 \times 10^{-4} \mathrm{~K}^{-1}$.
Significant nonlinearity of $n_{i}(T)$ is observed in the ICP.

The ICP-CP phase transition is accompanied with stepwise changes in $n_{i}: \delta n_{x}=2.1 \times 10^{-4}, \delta n_{y}=$ $2.4 \times 10^{-4}$ and $\delta n_{z}=1.9 \times 10^{-4}$. Thermal hysteresis $\sim 3 \mathrm{~K}$ is observed at heating. Note that all of these results are valid for the samples annealed for $8 \mathrm{~h}$. At the same time, jump-like changes of $n_{i}$ are not observed at the PT for the as-grown samples, both - in the cooling and heating runs (see Fig. 3, inset).

The annealing for $3 \mathrm{~h}$ leads to jump-like changes of $n_{i}$ at the PT: $\delta n_{x}=1.2 \times 10^{-4}, \delta n_{b y}=1.1 \times 10^{-4}$ and $\delta n_{z}=$ $0.8 \times 10^{-4}$. Notable dependence of the PT character and of the value of thermal hysteresis on the time of annealing suggests that the phase solitons are fixed by defects of the crystal lattice ("crystallization type" defects).

\section{Conclusions}

1. The spectral and temperature dependences of the refractive indices ni of free and mechanically clamped of the uniaxial stress along the principal crystallographic directions of $\mathrm{Rb}_{2} \mathrm{ZnCl}_{4}$ crystals were studied.

2. The increase of principal refractive indices under stress effect was observed, which is caused by the increase of bounds refraction and electron polarizability.

3. The uniaxial stress along the principal crystallographic directions mainly decreases an effective strength of UV oscillators and displaces an effective center of UV absorption band to the long wave region.

4. The annealing of samples leads to decrease of the magnitude of jump-like changes of ni at the phase transition. Its facts are due by influence the phase solitons are fixed by defects of the crystal lattice ("crystallization type" defects).

\section{References}

[1] S. Sawada, Y. Shiroishi, A. Yamamoto, M. Takashige, M. Matsuo, J. Phys. Soc. Japan 43, 2099 (1977).

[2] M. Wada, A. Sawada, Y. Ishibashi, J. Phys. Soc. Japan 47, 1185 (1979).

[3] H. Mashiyama, H.-G. Unruh, J. Phys. C, Solid State Phys. 16, 5009 (1983).

[4] K. Hamano, H. Sakata, K. Ema, J. Phys. Soc. Japan 54, 2021 (1985).

[5] V. Janovec, G. Godfroy, L.R. Godfroy, Ferroelectrics 53, 333 (1983).

[6] K. Hamano, Y. Ikeda, T. Fujimoito, K. Ema, S. Hirotsu, J. Phys. Soc. Japan 49, 2278 (1980).

[7] J. Kroupa, J. Fousek, Japan. J. Appl. Phys. 24, Suppl. 24-2, 787 (1985).

[8] P. Gunter, R. Sanctuary, F. Rohner, Phys. Status Solidi A 70, 583 (1982).

[9] S.V. Melnikova, A.T. Anistratov, Fiz. Tverd. Tela 25, 848 (1983). 